\title{
Formation of bubble chains over twin nozzles
}

\author{
Pawet Dzienis ${ }^{1, *}$, Romuald Mosdorf ${ }^{1}$, Daniel Tomaszuk ${ }^{1}$ and Wiotold Suchecki $^{2}$ \\ ${ }^{1}$ Bialystok University of Technology, Faculty of Mechanical Engineering, Wiejska 45C, 15-351 Białystok, Poland, \\ ${ }^{2}$ Warsaw University of Technology, Płock Campus, Institute of Mechanical Engineering, Department of Process Equipment, Ignacego \\ Łukasiewicza 17, 09-400 Płock, Poland
}

\begin{abstract}
In the paper the formation of bubble chains over two nozzles has been experimentally investigated and modelled. Bubbles were generated from twin brass nozzles (with the inner diameter equal to $1 \mathrm{~mm}$ ) to distilled water. The appearance and disappearance of alterative bubble departures have been investigated. It has been found that during disappearance of alternative bubble departures bubbles depart from nozzle outlets simultaneously. The PIV method was used to reconstruct the liquid velocity field around the bubble chains. The results of experiment were comparing with results of numerical simulation in which the Level Set method has been used. The PIV method show that during the alternate bubble departures the range of the velocity field formed by the bubble chain is smaller than in case when bubbles depart in nonalternative way. In this case the bubbles velocity above the nozzles outlet is greater in comparison with bubble velocity during the simultaneously bubble departures. The numerical simulations show that in alternative bubble departures bubble just after its departure is attracted to the common bubble chain. The hydrodynamic interactions between departing bubbles lead to uniform distribution of bubbles in bubble chain.
\end{abstract}

\section{Introduction}

The understanding of mechanisms associated with bubbles flow, bubble chains formation and bubbles interactions plays a major role in liquid mass transfer process [1]. The changes of liquid flow above the nozzle outlet modify bubble growth time and bubble waiting time. Nonlinear changes of boundary conditions (in liquid) of bubble growth lead to chaotic bubble departures [2].

When bubbles are departed from two neighbouring nozzles, growing bubbles interact between each other's by generation of liquid stream over the nozzles outlets. Hydrodynamic interactions between bubbles caused coalescence or bouncing of departing bubbles [3, 4, 5, 6]. Furthermore, in the paper [3], it was shown that the bubble coalescence is dependent on the liquid properties (such as viscosity and surface tension), distance between nozzles and the gas flow rate. Bouncing and coalescence of a pair bubbles generated from orifice and rising side by side, was investigated in the paper [4]. It was found that bubble occurrence of bubble bouncing or bubble coalescence process is connected with bubble Reynolds Weber and Morton number. In this paper the criteria of bubble coalescence for different numbers of those dimensionless numbers have been established. In the paper [5] it was founded that, bouncing process is associated with the competition between viscous and inertial effects, rather than to bubble shape deformation.
In the paper [6], the alternative bubble departure has been analysed. During the alternative bubble departures, bubble hydrodynamic interaction causes the formation of unique structure of bubbles flow above nozzle outlets [7]. Alternative bubble departures occur for selected air volume flow rates and distances between nozzles [6].

In present paper the liquid flow above nozzles during the appearance and disappearance of alternative bubbles departures have been investigated. In experimental investigations bubbles were generated from twin nozzles, to distilled water. The changes of pressure in gas supply system and videos contain the formations of bubbles were recorded simultaneously. Based on videos and time series of pressure fluctuations the ways of bubble departure were established. The Level Set and PIV method were used to determine to liquid flow above the nozzle during appearance and disappearance of alternative bubble departures.

\section{Experimental results}

During the experimental investigations bubbles were generated from twin brass nozzles (with inner diameter equal to $1 \mathrm{~mm}$ ) to distilled water. Schema of experimental setup has been shown in the Fig.1.

The air volume flow rate was measured using by flow meters - MEDSON s.c Sho-Rate-Europe Rev D, $\mathrm{P} 10412 \mathrm{~A}$. Using air valves, the air volume flow rates were changed in the range of $0.014 \mathrm{l} / \mathrm{min}$ to $0.125 \mathrm{l} / \mathrm{min}$.

*orresponding author: p.dzienis@pb.edu.pl 
During the experiment the distance between nozzles was equal to $4 \mathrm{~mm}$. Gas supply systems were powered by air pumps. The pressure of air, in gas supply systems, was set using a proportional pressure reducing valves Metalwork Regtronic. The adjustable pressure range is $0.05-10$ bar. In experimental setups the air pressure was set as 0.2 bar. The fluctuations of pressure were measured using by pressure sensors MPX.

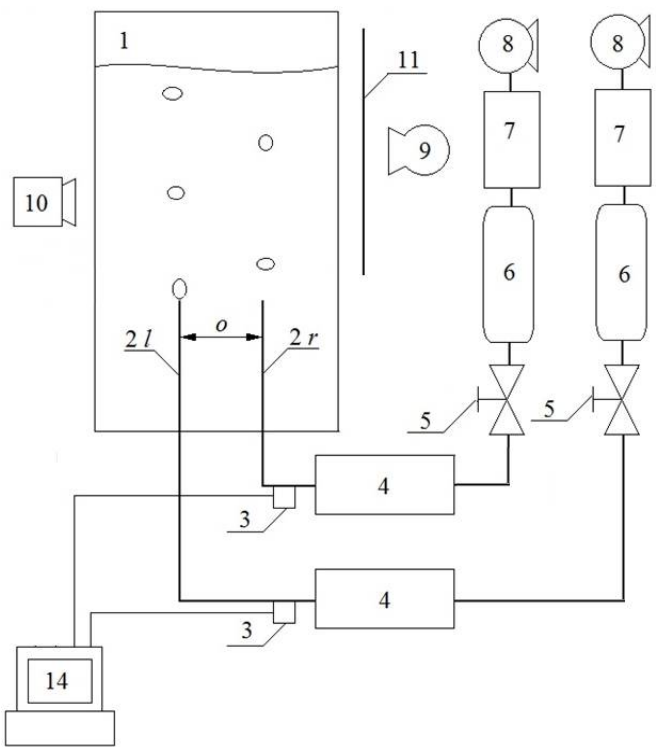

Fig. 1. Schema of experimental setup: 1 - glass tank, 2 - nozzles, 3 - pressure sensors, 4 - flowmeters, 5 - air valves, 6 - air tanks, 7 - pressure regulator, 8 - air pomp, 9 - linear laser, 10 - camera, 11 - computer acquisition system.

The air volume flow rate was measured using by flow meters - MEDSON s.c Sho-Rate-Europe Rev D, P10412A. Using air valves, the air volume flow rates were changed in the range of $0.014 \mathrm{l} / \mathrm{min}$ to $0.125 \mathrm{l} / \mathrm{min}$. During the experiment the distance between nozzles was equal to $4 \mathrm{~mm}$. Gas supply systems were powered by air pumps. The pressure of air, in gas supply systems, was set using a proportional pressure reducing valves Metalwork Regtronic. The adjustable pressure range is $0.05-10$ bar. In experimental setups the air pressure was set as 0.2 bar. The fluctuations of pressure were measured using by pressure sensors MPX.

The videos contain bubble chain formation have been recorded using high speed camera CASIO EX FX 1. The duration of videos was $45 \mathrm{~s}$. Videos have been recorded in grey scale with speed of 600 fps. Videos have been divided into frames. The example frames of videos have been presented in the Fig. 2 .

alternative bubble departures, b) alternative bubble departures.

In the Fig. $2 b$ the alternative bubble departures have been observed. The simultaneous bubble departures have been shown in Fig.2a. The disappearance of alternative bubble departures can be caused by disappearance of hydrodynamic interaction between moving bubble (departed from one nozzle) and bubble growing from neighbouring nozzle a)

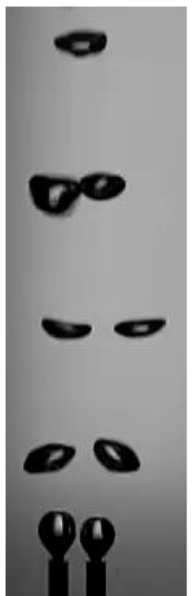

b)

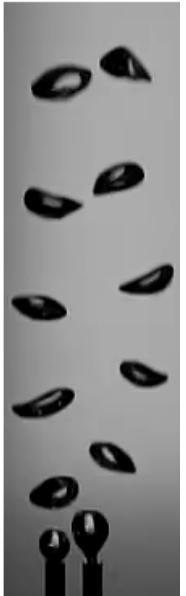

Fig. 2. Example frames of videos a) disappearance of disappearance of alternative bubble departures; b) alternative bubble departures.

In the Fig. 3 the results of PIV method analysis have been shown.

a)

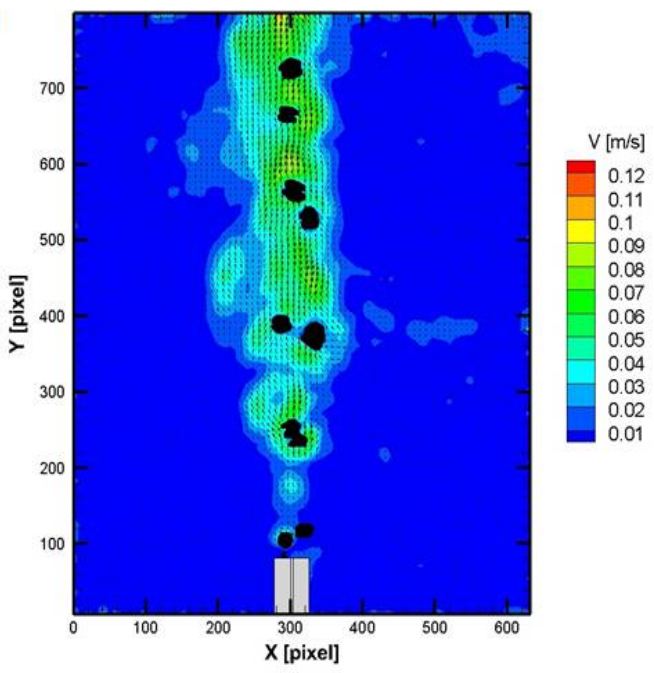

b)

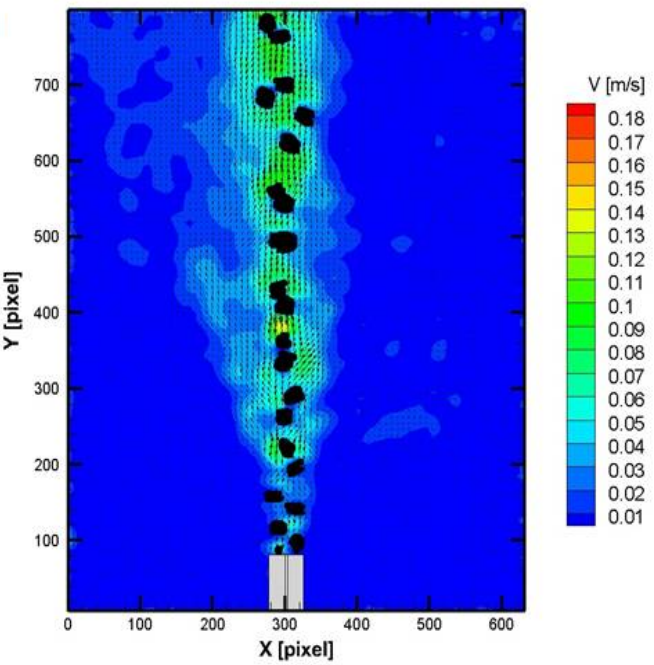

Fig. 3. Liquid flow above nozzle outlets, during alternative and simultaneous bubble departures. a) simultaneous bubble departures, b) alternative bubble departures. 


\section{Numerical analysis and results}

The model was based on level set method [8]. The Naviera-Stokes equations have been solved:

$$
\nabla \cdot \vec{u}=0
$$

$\rho \frac{\partial \vec{u}}{\partial t}+\rho(\vec{u} \cdot \nabla) \vec{u}=\nabla \cdot[-p I+\mu(\nabla \vec{u}+\nabla \vec{u} \mathrm{~T})]+\vec{F}+\rho \vec{g}+\sigma \kappa \delta \vec{n}$

where: $u$ - velocity, $g$ - gravity, $t$ - time, $I$ - identity matrix, $p$ - pressure, $\sigma$ - surface tension coefficient, $\kappa$ curvature, $n$ - the unit normal to the interface and $\delta$ Dirac delta function concentrated to the interface. Curvature $\kappa$, interface normal $n$ and Dirac delta $\delta$ :

$$
\begin{gathered}
\vec{n}=\frac{\nabla \phi}{|\nabla \phi|} \\
\kappa=\nabla\left(\frac{\nabla \phi}{|\nabla \phi|}\right) \\
\delta=6|\nabla \phi||\phi(1+\phi)|
\end{gathered}
$$

The level set function is smooth continuous function of $\phi$. Near the interface the function changes smoothly form 0 to 1 . Regions where $\phi<0.5$ represent the occurrence of liquid and regions where $\phi>0.5$ represent the occurrence of gas. The interface is represented by 0.5 contour of the function $\phi$.

The function $\phi$ is defined as follows [8]:

$$
\phi\left\{\begin{array}{cc}
>0.5 & \text { air } \\
0.5 & \text { int ererface } \\
<0.5 & \text { water }
\end{array}\right.
$$

The convection of the level set function is described by to the following equation:

$$
\frac{\partial \phi}{\partial \mathrm{t}}+v \cdot \nabla \phi=0
$$

The CFD Module of COMSOL Multiphysics ${ }^{\circledR}$ were used. In this model the following level-set equation was used:

$$
\frac{\partial \phi}{\partial \tau}+v \cdot \nabla \phi=\gamma \nabla \cdot\left(\varepsilon \nabla \phi-\phi(1-\phi) \frac{\nabla \phi}{|\nabla \phi|}\right)
$$

The left side of the equation defines the motion of the interface. The right side of the equation is responsible for numerical stabilization and reinitialization of the level set function. The parameter $\varepsilon$ controls the interface thickness and parameter $\gamma$ defines the intensity of reinitialization. The density and dynamic viscosity are described by the following formulas:

$$
\begin{aligned}
& \rho=\rho_{1}+\left(\rho_{2}-\rho_{1}\right) \phi \\
& \mu=\mu_{1}+\left(\mu_{2}-\mu_{1}\right) \phi
\end{aligned}
$$

Where: $\rho_{1}$ - liquid density, $\rho_{2}$ - gas density, $\mu_{1}$ liquid dynamic viscosity $\mu_{2}$ - gas dynamic viscosity.

Initial positions of bubbles for two ways of bubble departures have been shown in the Fig.4.

a)

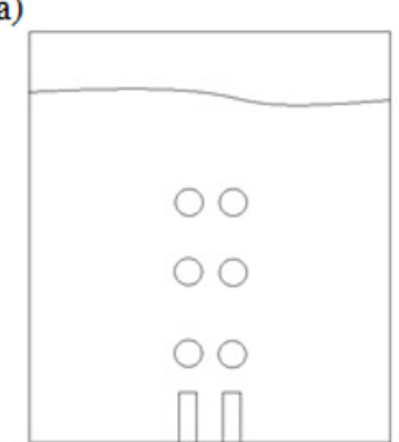

b)

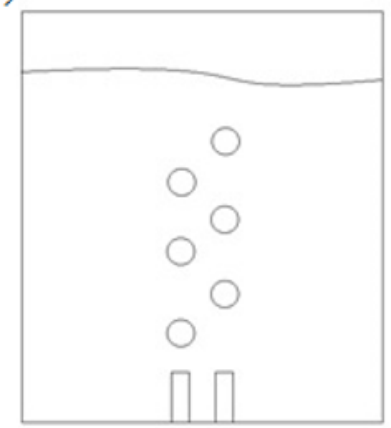

Figure 4

Fig. 4. Initial positions of bubble. a) simultaneous bubble departures, b) alternative bubble departures.

The diameters of bubbles were equal to $4.6 \mathrm{~mm}$. The results of numerical analysis where shown in the Fig.5. The time of analysis was equal to $1 \mathrm{~s}$. The presented structure of liquid flow above the nozzles outlets was obtained in $0.2 \mathrm{~s}$ of analysis.

In the Fig.6, vertical and horizontal components of liquid velocity above the nozzle outlets for alternative and disappearance of alternative bubble departures have been shown. 
a)

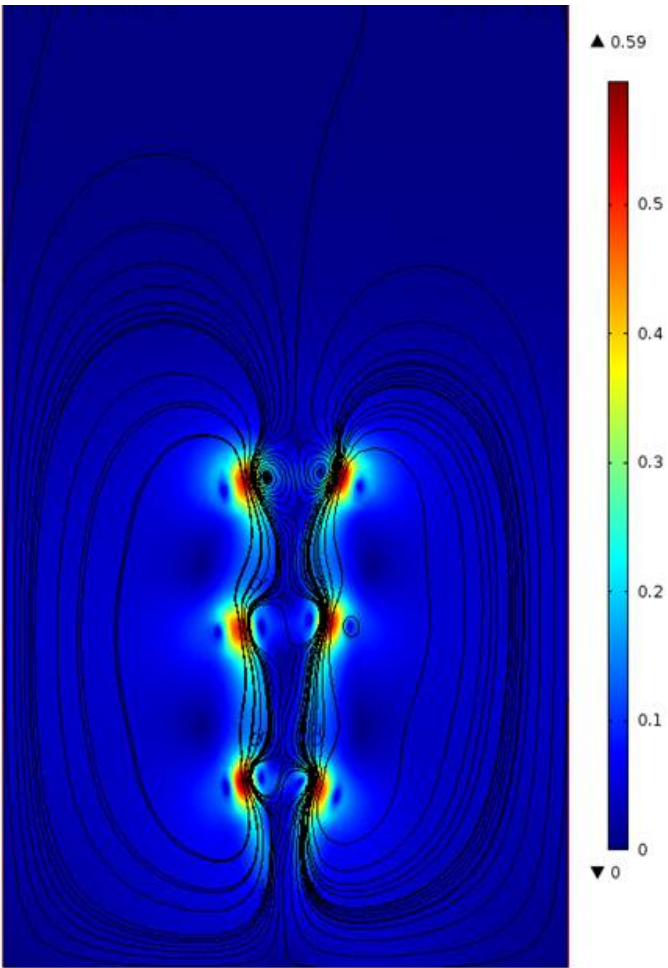

b)

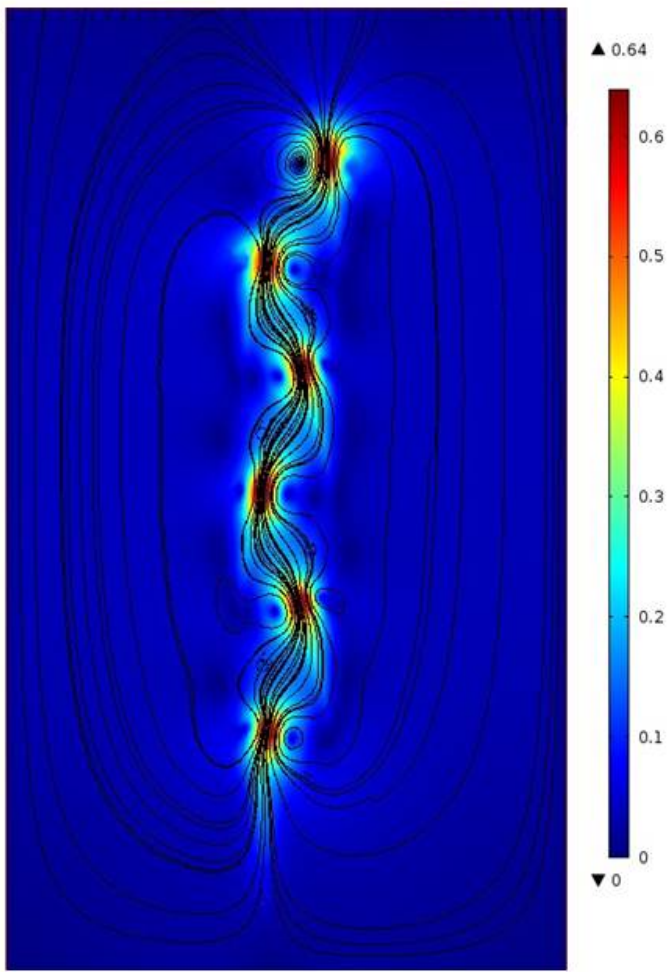

Fig. 5. Liquid streams formation above the nozzles outlets. a) disappearance of alternative bubble departures, b) alternative bubble departures.

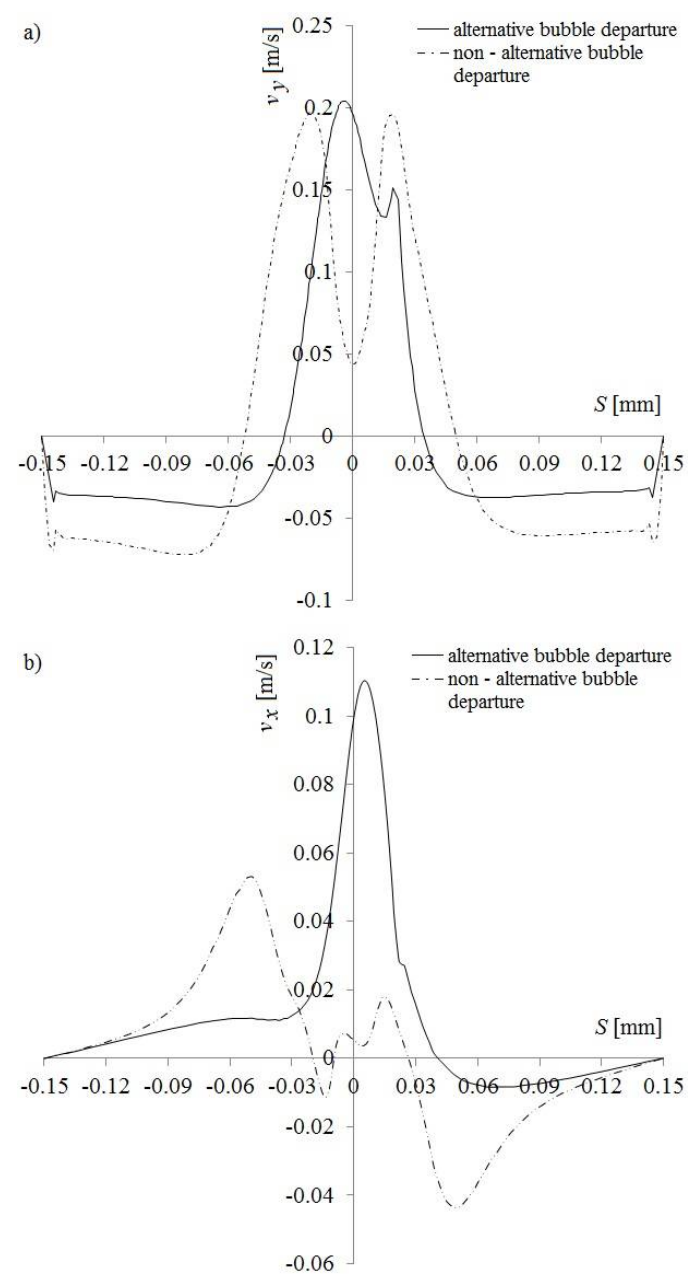

Fig. 6. Vertical and horizontal components of liquid velocity above the nozzle outlets for: alternative and simultaneous bubble departures. a) vertical $\left(v_{y}\right)$ liquid velocity component, b) horizontal $\left(v_{x}\right)$ liquid velocity component. The vertical and horizontal components of liquid velocity were calculated $2 \mathrm{~mm}$ above the nozzle outlets.

\section{Conclusions}

The results of experimental investigations and numerical simulations show that:

- during the alternative bubble departures the bubbles velocity above the nozzles outlet is greater in comparison with bubble velocity during the simultaneously bubble departures,

- during the alternative bubble departures the liquid flow generated by common bubble chain start just above nozzles outlet. In this case the liquid flow is closer to nozzles outlet in comparison with case when the bubbles depart in non-alternative way. Therefore in alternative bubble departures the growing bubble interacts with the liquid flow generated by previous bubble.

- numerical simulation shown that in alternative bubble departures bubble just after its departure is attracted to the common bubble chain.

- hydrodynamic interaction between departing bubbles leads to uniform distribution of bubbles in bubble chain, 
- the PIV method show that during the alternate bubble departures the range of the velocity field formed by the bubble chain is smaller than in case when bubbles depart in non-alternative way.

\section{Acknowledgments}

This work is part of the project no. S/WM/4/2017 conducted at the Faculty of Mechanical Engineering, Bialystok University of Technology.

\section{References}

1. M. Martin, J.M. Garcia, F.J. Montes, M.A. Galan, Chemical Engineering and Processing, 47, 17991809 (2008)

2. P. Dzienis, R. Mosdorf, Chemical Engineering Science, 109, 171-182 (2014)

3. T. Sanada, M. Watanabe, T. Fukano, Chemical Engineering Science 60, 5372-5384 (2005)

4. N.A. Kazakis, A.A. Mouza, S.V. Paras, Chemical Engineering Science, 63, 5160-5178 (2008)

5. T. Sanada, A. Sato, M.T. Shirota, M. Watanabe, Chemical Engineering Science 64, 2659-2671 (2009)

6. R. Mosdorf, T. Wyszkowski, International Journal of Heat and Mass Transfer, 61, 277-286 (2013)

7. R. Mosdorf, T. Wyszkowski, Int. J. Heat Mass Transfer, 54, 5060-5069 (2011)

8. S. Osher, J.A. Sethian, J. Comput. Phys. 79, 12-49 (1988) 